Service social

\title{
L'avenir-santé au féminin, sous la direction de Colette Gendron et Micheline Beauregard, Boucherville, Gaëtan Morin éditeur, 1989, 339 pages.
}

\section{Sylvie Berger}

Volume 39, numéro 2, 1990

Les problèmes sociaux

URI : https://id.erudit.org/iderudit/706493ar

DOI : https://doi.org/10.7202/706493ar

Aller au sommaire du numéro

Éditeur(s)

École de service social de l'Université Laval

ISSN

1708-1734 (numérique)

Découvrir la revue

Citer ce compte rendu

Berger, S. (1990). Compte rendu de [L'avenir-santé au féminin, sous la direction de Colette Gendron et Micheline Beauregard, Boucherville, Gaëtan Morin éditeur, 1989, 339 pages.] Service social, 39(2), 249-251.

https://doi.org/10.7202/706493ar d'utilisation que vous pouvez consulter en ligne.

https://apropos.erudit.org/fr/usagers/politique-dutilisation/ 
tale et universelle pour convenir à chacun. D'autre part, la présente étude est davantage conçue afin de nourrir la réflexion personnelle de quiconque désire logiquement assumer le processus de son propre vieillissement.

Tout de suite, on aborde un ouvrage de base, un manuel sobre qui cherche à définir et à clarifier des données d'importance capitale. Pour en arriver à ses fins, le gérontologue partage son exposé en sept tranches ou chapitres, lesquels s'imbriquent et assurent une connaissance évolutive d'un phénomène irréversible, mais qu'on peut apprivoiser, voire en atténuer certains effets. $\mathrm{D}^{\prime}$ abord, on présente la gérontologie comme étant objet de réflexions existentielles, collectives et scientifiques. On s'attache au vieillissement dans ses définitions, son expérience, sa crise de l'existence. On perçoit "l'atteinte de l'intégrité " selon une approche négative ou positive; après l'avoir définie, on dira sa manifestation et ses conditions. On traite de la crise d'identité sous ses multiples facettes pour passer ensuite à celle d'autonomie : manifestations, pertes et causes. On discute du phénomène d'appartenance et on retient ce que peut être : la fonction sociale des stéréotypes de la vieillesse, le retrait du courant de la vie, l'ennui et la solitude, sans oublier l'aptitude à s'intéresser à la vie. Enfin, on parle d'une relation d'aide spécifique et d'un lieu d'expression de soi, en invitant à faire place à l'inattendu pour arriver à dépasser les limites habituelles de l'expression. Tout cela et encore, dans une lecture pondérée, raisonnée, où l'on perçoit la formation de l'auteur aux méthodes des sciences sociales, de la philosophie et de I'histoire.

$\mathrm{Ne}$ cherchons pas ici un étalage de subtils " distinguos". Le tout est élémentaire et nécessaire au sens bénéfique et formel des termes, et c'est ce qui constitue sa bienvenue. Travailleurs sociaux, psychologues, intervenants " chevronnés " auprès des gérontins se rappelleront certes des données déjà mémorisées, compte tenu de la progression constante de l'art et de la science gérontologiques. Néanmoins, et c'est cela qui s'offre hautement et particulièrement valable : l'étudiant et l'étudiante en sciences humaines doivent de prime abord se familiariser avec cette "Introduction", - tout comme le citoyen qui marche de l'avant - soucieux de vivre et non pas "subir sa vieillesse". aloi.

Au rythme de son pas, le lecteur rencontre ici des ressources de bien bon

Louis-Roland Paradis
Université du Québec
Trois-Rivières

L'avenir-santé au féminin, sous la direction de Colette GENDRON et Micheline BEAUREGARD, Boucherville, Gaëtan Morin éditeur, 1989, 339 pages.

L'avenir-santé au féminin réunit quatorze auteurs et dix-sept textes. Ce recueil se veut le prolongement d'un premier ouvrage publié sous la même direction en 1985 : Les Femmes et la santé. La majorité des textes offerts (onze) traite de sujets liés étroitement à la fonction reproductrice des femmes; les autres abordent des thèmes variés tels que l'alimentation, la violence conjugale, etc. À la fin de chaque texte, on retrouve des références bibliographiques suffisamment récentes et diversifiées. 
De formation professionnelle différente, mais ayant toutes deux une approche féministe, les auteures constatent et nous forcent à ne pas oublier que, aujourd'hui plus qu'hier, les femmes sont les plus grandes utilisatrices des services de santé et qu'elles ont un indice de santé global moins bon que celui des hommes. En effet, on apprend que, même si aujourd'hui encore les femmes vivent plus longtemps que les hommes, elles passent le quart de leur vie avec un problème de santé handicapant. On note que l'obésité les atteint davantage, qu'elles font moins d'activité physique et souffrent plus de détresse psychologique.

J'ai pa relever quelques constatations générales omniprésentes tout au long de l'ouvrage, regroupées ici sous quatre items.

\section{1 - Système médical}

Les auteures remettent en question, à plusieurs reprises, le pouvoir médical et la tangente prise par la médecine moderne. Alors que celle-ci devrait être au service du public, elle suit ses propres intérêts, sa propre trajectoire. On assiste à une surmédicalisation d'événements naturels, entre autres la grossesse et l'accouchement. La recherche médicale se fait sans orientation, sans priorité axée sur les besoins des femmes, pour obéir à des modes du moment ou pour la gloire du chercheur (ex. recherches coûteuses sur les nouvelles technologies de reproduction) alors que peu de recherches sont entreprises en prévention de l'infertilité et sur la ménopause. Les médecins sont perçus comme des interventionnistes (ex. hystérectomie, médication) et peu enclins à informer leurs clientes de façon à ce qu'elles puissent prendre une décision éclairée (ex. méthodes diagnostiques lors d'une grossesse, césarienne, tranquillisants, etc.).

\section{2 - Établissement des priorités}

Les priorités pour la santé des femmes sont encore à établir au Québec. On peut cependant se référer à de récentes consultations de la base, en Australie, qui ont permis d'établir dix priorités pouvant être facilement transposées dans notre réalité québécoise. On mentionne que rien ne pourra remplacer une large consultation de la base auprès des femmes sur leurs besoins dans le domaine de la santé.

\section{3 - Attitude des femmes envers la santé et le monde médical}

Dans les différents textes, on signale que les femmes elles-mêmes ont une attitude ambivalente face au monde médical. Elles seraient plus ou moins prisonnières de leur peur (peur que le bébé soit anormal, peur de souffrir, etc.) et tenues de faire confiance par manque de connaissances et d'informations. Dans plusieurs situations concernant leur santé (grossesse, avortement, ménopause, hystérectomie, infertilité, etc.), les femmes demeurent trop souvent passives, à la merci des décideurs, leur corps étant ainsi aliéné.

\section{4 - Pour un avenir en santé}

II se dégage de plusieurs textes que la santé des femmes passe par la formation des intervenants, un changement d'attitude du monde médical et 
même une diminution du pouvoir excessif de ce dernier. On demande aussi la possibilité de bénéficier d'approches non médicales, de centres de santé pour les femmes, de thérapies féministes. Les femmes elles-mêmes devraient changer leurs habitudes de vie, apprendre à se mobiliser et à aller chercher l'information.

Cet ouvrage présente un constat de la situation de santé actuelle des Québécoises, vue sous divers angles par des femmes reconnues dans leur domaine respectif. Fait à noter : bien qu'on assiste de plus en plus à une féminisation de la profession médicale, on ne retrouve ici aucun article écrit par l'une de ces femmes. De même, il aurait sans doute été intéressant de lire les attentes, en matière de santé, des utilisatrices elles-mêmes. II me vient aussi un certain questionnement face au choix ou plutôt au non-choix des thèmes; par exemple, on passe à peu près sous silence les problèmes de santé mentale, les MTS, les maladies liées au vieillissement, la fatigue des femmes, I'usage des drogues et de l'alcool, les agressions sexuelles et incestueuses, etc. Cependant, pour les sujets traités et pour les avenues futures, les intervenants et intervenantes gagneront à la lecture de cette publication : les hommes, pour mieux comprendre, et les femmes, pour se souvenir que les acquis, dans ce domaine comme ailleurs, sont toujours fragiles et surtout rarement gratuits.

Sylvie Berger

Infirmière-chef, Département d'urgence

Centre hospitalier de l'Université Laval

\section{Prison et ordre social au Québec, par Jacques LAPLANTE, Ottawa, Les Presses de I'Université d'Ottawa, 1989, 238 pages.}

Il s'agit d'un ouvrage publié à la suite d'une recherche universitaire qui se proposait d'étudier le système de justice pénale en milieu québécois et plus particulièrement d'examiner l'institution de la prison comme moyen de répression des pauvres, couche sociale défavorisée et surreprésentée dans les milieux carcéraux.

À cette fin, I'auteur a rédigé sa publication en trois parties, qui correspondent à toutes fins pratiques à trois périodes significatives de l'histoire du Québec. La première partie, qui couvre la période 1608-1830, explique de façon détaillée le fonctionnement naturel de la justice autochtone des Amérindiens, puis l'imposition successive des mesures pénales sous les régimes français et anglais.

Dans la seconde partie, correspondant à la période 1830-1930, l'auteur tente de démontrer comment $s^{\prime}$ est progressivement construit le système pénal et à quel point la prison est devenue un moyen organisé pour réprimer la population cible des pauvres au détriment des autres couches sociales.

Enfin, dans la troisième et dernière partie couvrant la période de 1930 à nos jours, I'ouvrage analyse la phase de consolidation du système, le mouvement des réformes et les finalités thérapeutiques introduites dans le discours pour justifier le maintien et le développement de la prison, au nom de l'ordre et de la défense sociale.

Pour guider sa démarche d'analyse tout au long de l'ouvrage, l'auteur fait appel à des paramètres qu'il nomme "les états fondamentaux de comman- 